\title{
Psicopatología de la ipseidad y la corporalidad: a propósito de un caso de esquizofrenia cenestopática.
}

Psychopathology of ipseity and embodiment: apropos of a case of cenesthopathic schizophrenia.

Rubén Valle 1,2,a , Milko Di Natale-Guevara ${ }^{2,3, a}$, Lizardo Cruzado ${ }^{2, b, 3, c, c}$

\section{RESUMEN}

La fenomenología ha dividido conceptualmente al cuerpo humano en el "cuerpo-objeto", la entidad física que puede ser percibida por uno mismo y por otros, y en el "cuerpo-vivido", que es la experiencia inmediata del propio cuerpo, tácitamente experimentado (o vivido) en una perspectiva de primera persona. El "cuerpo-vivido" origina la experiencia pre-reflexiva de la vida, adscribible al yo mínimo, core self o ipseidad que constituye el sustento nuclear de la conciencia del yo. Este modo corporal opera implícitamente en cada acción que ejecutamos en el mundo y nos permite la aprehensión y entendimiento del entorno. Sin embargo, en algunos procesos mórbidos el cuerpo puede perder su calidad de "transparencia" y volverse anormalmente explícito, tal como ocurre en la esquizofrenia. En esta patología, los pacientes sufren un distanciamiento subjetivo entre la mente y el cuerpo al que se denomina descorporalización y, como consecuencia, experimentan un debilitamiento del sentido básico del yo, alteraciones de la relación sujeto-objeto y del otorgamiento de significados, así como en la vivencia de la intersubjetividad. Anormalidades del "cuerpo-vivido" han sido identificadas previamente en los casos de esquizofrenia desorganizada, paranoide y catatónica. En este reporte analizamos cómo se manifiestan las alteraciones del cuerpo-vivido y de la ipseidad en un caso de esquizofrenia cenestopática.

PALABRAS CLAVE: Esquizofrenia, cuerpo humano, estado de conciencia, psicopatología. (Fuente: DeCS BIREME).

\section{SUMMARY}

Phenomenology has conceptually divided the body into the "object-body", the physical entity that can be perceived by oneself and others, and the "living-body", that is the immediate experience of the body, tacitly experienced (or lived) in a first-person perspective. The "living-body" originates the pre-reflective experience of life, also ascribable to the minimal self, core self or ipseity, which constitutes the nuclear support of self-consciousness. This body mode implicitly operates in every action that we execute in the world, and allows us to grasp and understand the environment. However, in some morbid processes, the body may lose its quality of "transparency" and become anomalously explicit, as it occurs in schizophrenia. In this disorder, patients suffer a subjective estrangement between mind and body, which is called decorporalization or disembodiment, as a consequence of which, the patients experience a weakness of the basic sense of self, abnormalities of the subject-object relationship and alterations

Facultad de Medicina de San Fernando, Universidad Nacional Mayor de San Marcos. Lima, Perú.

2 Instituto Nacional de Salud Mental Honorio Delgado-Hideyo Noguchi. Lima, Perú.

3 Facultad de Medicina Alberto Hurtado, Universidad Peruana Cayetano Heredia. Lima, Perú.

a Médico residente de Psiquiatría; ${ }^{b}$ Psiquiatra; ${ }^{\text {c }}$ Profesor auxiliar. 
Psicopatología de la ipseidad y la corporalidad: a propósito de un caso de esquizofrenia cenestopática.

in meaning-bestowing, as well as in the experience of intersubjectivity. Abnormalities of the "living-body" have previously been identified in cases of disorganized, paranoid and catatonic schizophrenia. In this report we analyze how the disorders of "living-body" and ipseity are manifested in a case of cenesthopathic schizophrenia.

KEYWORDS:Schizophrenia, human body, consciousness, psychopathology. (Source: MESH NLM)

\section{INTRODUCCIÓN}

La esquizofrenia es, ante todo, un trastorno del sujeto y de la subjetividad y así, de la experiencia de sí mismo y del mundo $(1,2)$. Aunque las vivencias anómalas de la conciencia del yo fueron descritas en la psicopatología clásica (Kraepelin, Bleuler, Jaspers y K. Schneider, entre otros), éstas no son recogidas específicamente en los criterios diagnósticos de las clasificaciones actuales (2). Sin embargo, la relevancia clínica y su valor potencial para el diagnóstico han resurgido en la época reciente por el interés en la detección de los estadios tempranos de la esquizofrenia (3).

Dado que se trata de un constructo, no existe una definición inequívoca del self o el yo ni hay consenso absoluto sobre la terminología empleada en su descripción (4). En las investigaciones fenomenológicas más recientes-obviamente hablamos de fenomenología del hemisferio occidental-, el desarreglo primario de la psicopatología de la esquizofrenia se postula a nivel del yo mínimo, core self, o ipseidad -es decir, la base elemental del yo como sujeto de experiencia y agente de acción-. Igualmente, se asume que la perspectiva del yo mínimo tiene como requisito esencial la vivencia de la corporalidad (embodiment) $(5,6)$, en tanto organizadora de la experiencia a partir de las capacidades vivenciales de la motilidad y la percepción. Por tanto, el disturbio de la corporalidad (disembodiment) será sustento explicativo de múltiples alteraciones de la ipseidad y de experiencias patológicas en la esquizofrenia $(7,8)$, como se precisará más adelante.

El cuerpo es el instrumento mediante el cual el individuo se comunica e interactúa con el mundo: el cuerpo entendido como interjuego psicofísico, fluido y constante, entre el cuerpo vivido (Leib) y el cuerpo físico (Korper) (3). El "cuerpo-objeto" es el cuerpo investigado desde afuera, es el cuerpo de la anatomía y la fisiología que puede ser medido y examinado mediante instrumentación biomédica $(7,9)$, mientras que el "cuerpo-vivido" es el cuerpo experimentado desde adentro, es la experiencia que un sujeto tiene de su cuerpo en una perspectiva de primera persona
(3). Normalmente, el cuerpo se mantiene circunscrito a una dimensión tácita, no plenamente consciente, y opera implícitamente en la configuración de la percepción del ambiente externo (10). Sin embargo, en la esquizofrenia, el cuerpo pierde su "transparencia", su condición tácita, y se torna explícito: existe un chocante desplazamiento al predominio de la vivencia del cuerpo como objeto, esto es, el cuerpo físico, con las secuelas consiguientes: vivencias de alienación e imposición, despersonalización y desrealización y, finalmente, hasta la emergencia de alucinaciones cenestopáticas, que representan el distanciamiento y disyunción entre el yo y el cuerpo (11). La esquizofrenia resulta siendo así, por excelencia, la patología en la que el individuo sufre una pérdida de la "transparencia corporal" $(8,10)$.

Estudios previos han analizado las alteraciones del cuerpo-vivido en casos de esquizofrenia desorganizada, paranoide y catatónica y se ha verificado que alteraciones de la corporalidad y la ipseidad estaban presentes en cada una de estas patologías, incluso desde etapas tempranas de la enfermedad (10). En el presente artículo, presentamos el caso de una paciente diagnosticada de esquizofrenia cenestopática y analizamos cómo los aspectos fenomenológicos de la ipseidad y la corporalidad se ven afectados en esta patología.

\section{Caso Clínico}

Paciente mujer de 24 años, soltera, con secundaria completa, de religión evangélica y sin ocupación. A la edad de 12 años empezó a experimentar una excesiva preocupación por el desarrollo de sus caracteres sexuales secundarios pues no quería crecer ni madurar físicamente. Con ese fin, presionaba sus senos para que no protruyan, se arrancaba el vello púbico y ejercía presión sobre sus brazos y muslos para tenerlos más delgados. Además deseaba que su cuello fuese más corto y ancho, sus piernas más prominentes en la parte posterior y las muñecas y los pies más finos. Realizaba estas maniobras alrededor de cuatro horas al día y llegó a causarse múltiples equimosis en su cuerpo. De hecho, ya desde pequeña se había sentido diferente a sus hermanas (en la complexión, algunas 
zonas corporales y algo más que ella misma no podía definir). A los 14 años de edad, la paciente empezó a sentir que era observada y que los transeúntes en la calle se burlaban de lo fea que ella era. Por eso evitaba salir de su casa, se aisló y tornó distante con su familia. Seguía presionando diversas partes de su cuerpo y se sentía inconforme con sus formas femeninas. Realizaba escasas actividades domésticas y dejó de asistir al colegio.

A los 20 años de edad, la paciente empezó a sentir que su cabeza "se introducía" en su tórax, como si su cuello se encogiese, y también que sus hombros y muslos se acortaban; asimismo experimentaba la sensación de que dentro de su boca sus dientes se apiñaban entre sí. Se quejaba de que como producto de la presión que había ejercido sobre distintas partes de su cuerpo a lo largo del tiempo, ahora tenía la cara más larga, el cuello más ancho, los senos más grandes, los ojos más redondos y las mejillas caídas. Concomitantemente, la paciente tuvo la certeza de que podía mover "hacia adentro" diferentes partes de su cuerpo. Por ejemplo, creía que podía introducir un brazo dentro de su tórax y luego volver a sacarlo cuando ella quisiese. Sin embargo, no comunicó a nadie sobre estos fenómenos. A los 22 años, sus familiares la llevaron por primera vez a la consulta psiquiátrica por notarla muy desaliñada, irritable, triste y aislada socialmente. Fue diagnosticada de depresión con síntomas psicóticos y recibió tratamiento con sertralina, risperidona y clonazepan. El tratamiento fue cumplido de manera irregular, las alucinaciones cenestésicas y las delusiones somáticas persistieron y solo se apreció una leve mejoría en el estado de ánimo.

A los 23 años, la paciente empezó a presentar alucinaciones auditivas. Esporádicamente escuchaba la voz de su hermana que jugaba con su sobrino y la risa de éste. A esa edad la paciente tuvo su primer intento suicida tomando diez pastillas de clozapina (pertenecientes a su hermano, diagnosticado con esquizofrenia paranoide) luego de presentar alucinaciones auditivas que le ordenaron que se quite la vida. Fue llevada a emergencia, donde permaneció en observación por cuatro días. En las evaluaciones posteriores, la paciente comentó por primera vez sus molestias corporales, las que en ese momento consistían en que, a su juicio, tenía la boca deforme, los pómulos prominentes y los surcos nasogenianos muy marcados: se miraba constantemente en el espejo y "no se reconocía". También se quejaba de sentir muy pesados sus pies y su cabeza y afirmaba que sus extremidades superiores se elongaban a voluntad, además explicó que levantaba las cejas para que su cabello oculte una cicatriz de su frente y así había ocasionado que su cabeza se estire y cambie de forma. Fue diagnosticada de esquizofrenia cenestopática y recibió tratamiento con risperidona, que le produjo marcado parkinsonismo; el cambio a quetiapina tampoco fue tolerado por excesiva somnolencia. Posteriormente, la paciente decidió reemplazar el tratamiento farmacológico por uno "naturista" y empezó a asistir a la iglesia más frecuentemente.

Luego de esto, el cuadro clínico se intensificó, con recrudecimiento de las alucinaciones auditivas y cenestopáticas: se sentía agobiada pues en un momento cuando observó un objeto de cerca, advirtió que sus ojos se habían quedado como "pegados a su nariz" y sentía que la sangre no podía circular en su cabeza. Ante esta vivencia desasosegante, la paciente intentó suicidarse tomando nuevamente una sobredosis de clozapina, por lo que fue llevada a emergencia y quedó en observación. Tras su alta, se inició tratamiento farmacológico, esta vez con risperidona y clozapina, encontrándose buena repuesta clínica y tolerabilidad con ambos medicamentos a bajas dosis. En esta ocasión hubo regularidad con la prescripción farmacológica y, al año de seguimiento, la paciente se mantenía clínicamente estable, eutímica, sin delusiones y sin cenestopatías. Asimismo, había mejorado su aliño, apoyaba en las labores de la casa y no tenía problemas en salir a la calle.

\section{DISCUSIÓN}

La configuración fenomenológica del yo se estructura en tres niveles. El núcleo básico y puntual del yo, denominado "yo mínimo", "core self" o ipseidad -del latín ipse, que significa "sí mismo"-, involucra la vivencia inmediata y pre-reflexiva del existir, en donde el que siente y lo que es sentido constituyen el mismo ente. Este nivel es el requisito previo y básico para toda otra experiencia pues sustenta la conciencia implícita, tácita e indubitable de lo que es $m i$ experiencia (1). El segundo nivel es el yo reflexivo y que se apoya en la ipseidad. Es el yo que describe la psicopatología clásica en el acápite de la conciencia del yo, con sus cualidades de unidad, identidad, sentimiento de actividad y demarcación del yo en oposición a lo externo. Es un yo poseedor ya de la capacidad de ser reflexivo y consciente de sí mismo (12). Y, como supraestructura, se establece el tercer nivel que es el yo narrativo, yo social o yo biográfico y que abarca los componentes de identidad social, historia biográfica, personalidad, hábitos, autoestima, 
y es el ámbito donde se despliegan los procesos metacognitivos (1).

Por otro lado, la corporalidad está constituida por la experiencia del cuerpo vivido, sin conciencia reflexiva ni requisito de monitorización perceptiva: es el trasfondo de experiencia corporal inherente a la vivencia del yo primario (3). Esta autoconciencia primigenia posee naturaleza eminentemente sensorial y propioceptiva. Las relaciones entre el yo mínimo y la vivencia de la corporalidad son íntimas y complejas $(6,7)$ (gráfico 1). Este vínculo puede explicarse en la medida en que todas las vivencias a nivel de la ipseidad, es decir, en la perspectiva de primera persona, ineludiblemente requieren de la corporeidad del yo, por ser condición en la que arraigan el movimiento y la percepción, los que, por sí mismos, instauran un espacio egocéntrico primario $(5,12)$. Es decir, el mundo se presenta ante el yo nuclear gracias a la dimensión y potencia espacial que brinda el cuerpo a su yo poseedor.

Se han descrito las aberraciones secundarias a la falla de la corporalidad per se $(6,7)$, y asimismo se han catalogado los desarreglos psicopatológicos propios de la ipseidad $(5,13)$. El modelo paradigmático de las alteraciones del yo en la esquizofrenia actualmente es el de Sass y Parnas, que compendia aportes de Minkowski, Jaspers, Blankenburg y Conrad (tabla 1) (12). En el análisis de nuestro caso, recogemos además la clasificación efectuada por Stanghellini de las perturbaciones de la corporalidad en base a la influencia que ejercen sobre el yo mínimo o nuclear (6), aunque como es obvio, dada la naturaleza forzadamente dual cuerpo-mente del pensamiento occidental, existe amplia correspondencia entre uno y otro ámbito (corporalidad / ipseidad, como caras opuestas pero inseparables de una misma moneda). A nivel operativo, el instrumento que recoge ampliamente las alteraciones del yo en la esquizofrenia es el EASE (Examination of Anomalous Self-Experience) (14), y que es fuente de prolífica investigación empírica en la actualidad. Los agrupamientos de fenómenos descritos en el EASE son los vinculados a: cognición y flujo de conciencia, conciencia de sí mismo y presencia, fenómenos de la experiencia corporal, demarcación del yo y transitivismo y, finalmente, reorientación existencial. Como puede notarse, las experiencias anómalas del yo que con este instrumento se recogen, involucran tanto vivencias psíquicas como vivencias corporales en su interfase más íntima y nuclear.

Según Stanghellini, la vivencia de la corporalidad, al sufrir perturbaciones, redundará sobre la ipseidad; en primer lugar, en la falla de la vivencia de posesión de la experiencia (mineness, en inglés) y de agencia de la experiencia, es decir, la vivencia de ser el yo el ejecutor de los actos de la conciencia. Esta "crisis" de la ipseidad origina un yo incapaz de vivir sus contenidos mentales como propios y una desvitalización del yo (falta de presencia), que se experimenta entonces como un yo vacío o hasta como un simple e inerme espectador de sus propias percepciones, pensamientos y actos, de los que ya no se siente ejecutor ni protagonista (11).

Gráfico 1. Tres dimensiones de la experiencia del cuerpo vivido.

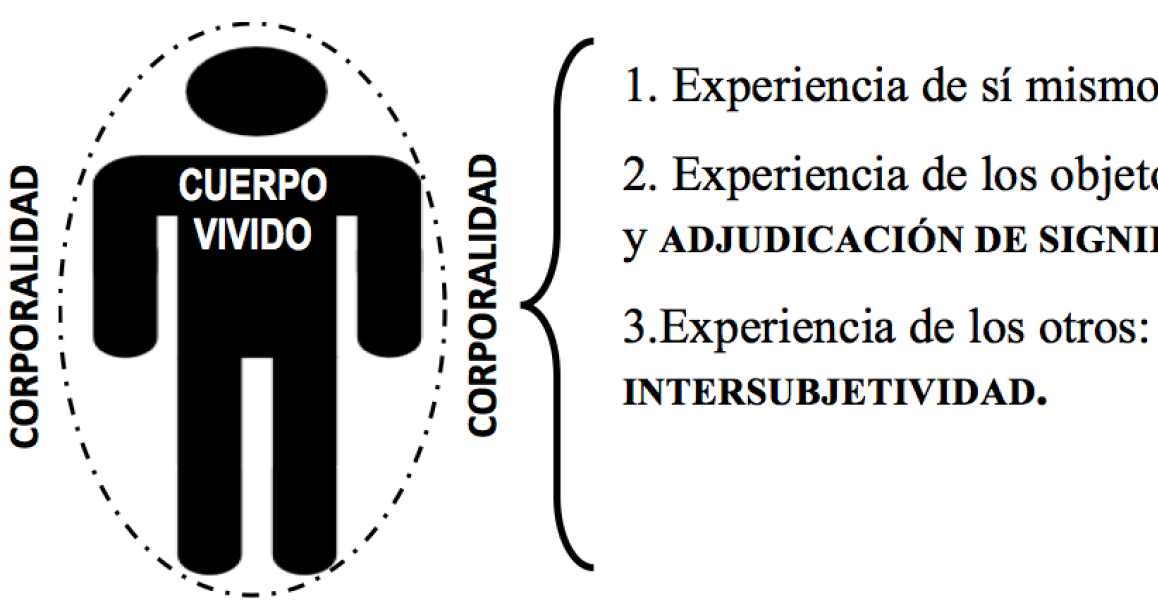


Pero además, la corporeidad normal sustenta las relaciones del yo con los objetos y permite lograr la conciencia de los mismos y otorgar significados a la percepción de ellos. El cuerpo vivido es fuente de comprensión constante de todos los objetos con los que interactúa, a través de la cinestesia y todas las aferencias sensoriales que constantemente se reciben del entorno en que el yo se halla sumergido $(11,12)$. Literalmente, el cuerpo "aprehende" el significado de los objetos del entorno en la medida en que interactúa con ellos y capta su inherente capacidad de ser manipulados. Esto dota al ambiente aprehendido por el yo de un "sentido natural de las cosas" que, al desvanecerse, priva a los objetos de su significado ordinario. Así, surgen nuevos significados, de naturaleza peregrina e idiosincrática (verbigracia, como en las delusiones perceptivas) que propician una resignificación del entorno e incluso distorsionan las mismas expresiones idiomáticas que designan a los objetos. Las palabras llegan a ser despojadas de su significado original y se usan para crear significados heteróclitos que se adjudican a los entes de un entorno trastocado y aberrante $(9,10)$.

Finalmente, y siempre según Stanghellini, la descorporalización se refleja en el ámbito de la intersubjetividad a través de la despersonalización autopsíquica y somatopsíquica que dificultan la sintonía corporal entre el yo propio y el de los otros individuos. De este modo, el autismo esquizofrénico puede ser una huida protectora a esta incapacidad de empatía con los otros y remata en el aislamiento social característico de la enfermedad, pues el mundo social, en vez de verse como una red preñada de afectos y emociones, se presenta amenazante, frío, excluyente e incomprensible $(10,11)$. Todas estas alteraciones se compendian en la seminal concepción de Minkowski de la "pérdida del contacto vital con la realidad" (15). En la tabla 2 se resumen las alteraciones de la corporalidad y su influjo sobre ámbitos del yo mínimo, ilustradas con viñetas clínicas de nuestro caso, y en la tabla 3 se enumeran las alteraciones del cuerpo vivido, ejemplificadas con igualmente con expresiones de nuestra paciente.

En cuanto a los hallazgos neurobiológicos subyacentes a los disturbios de la ipseidad, $(16,17)$ se ha apuntado a fallas de conectividad neuronal entre áreas dedicadas a la integración intra e intermodal de las aferencias sensoriales y las eferencias motoras, esto es, a nivel de la unión témporo-parietal derecha, el área parietal inferior y el córtex parietal posterior, además de la ínsula, y también la corteza prefontal medial y lateral. Estos últimos circuitos dan el correlato de emociones complejas y deseos a la vivencia primaria del yo.Se ha descrito asimismo, en la corteza insular del hemisferio no dominante, el sustrato neuronal para la evaluación de cómo el individuo se siente: este sistema, no hallado en otros primates, informaría no solo de la homeostasis visceral sino de la condición fisiológica del individuo y sería el primordio de la conciencia del yo. Se ha comprobado recientemente irrefutables anomalías de la interocepción en pacientes con esquizofrenia (18). Además, en exámenes neuropsicológicos e imagenológicos, es muy sugerente encontrar que áreas específicas del córtex cerebral occipital se activan ante la visión del propio

Tabla 1. Alteraciones psicopatológicas de la ipseidad. (12)

$\frac{\text { ALteraciones DE LA IPSEIDAD }}{\text { IPSEIDAD }}$

Vivencia íntima, empírica y primaria de constituir un ser vivo y sujeto de experiencia con perspectiva de primera persona (yo) en el mundo.

\section{Abolición del sentimiento de presencia de sí mismo}

Disminución de la intensidad o vitalidad de la propia presencia del yo.

- Abolición del sentimiento de posesión (de ser dueño de la experiencia).

- Abolición del sentimiento de agencia (de ser el actor de la vivencia).

\section{Hiperreflexividad}

Conciencia incrementada de aspectos de la experiencia normalmente tácitos. Puede ser secundaria a lo anterior (compensadora ante pérdida de la evidencia natural de yo).

\section{Alteración de la aprehensión (“hold / grip”)}

Pérdida de contacto vital con la realidad, alteración de la configuración normal del campo de la conciencia y de la aprehensión de los objetos del entorno por el abatimiento de la prominencia o estabilidad con que dichos objetos asoman a la conciencia. 
Psicopatología de la ipseidad y la corporalidad: a propósito de un caso de esquizofrenia cenestopática.

Tabla 2. Definición de las alteraciones secundarias a la descorporalización y ejemplos ilustrativos del caso clínico. (6)

DESCORPORALIZACIÓn DEL Yo

Sentimiento general de existir como ajeno al mundo, de pérdida de presencia en el mundo. El individuo se siente como separado de sus acciones y de sus experiencias. El cuerpo es privado de la posibilidad de vivir experiencias personales como propias: el individuo es simple espectador de sus propias percepciones, acciones y pensamientos. Vivencias de imposición y pasividad somática.
- "De pronto surgía algo fuerte dentro de mí que me obligaba a que me frote el cuerpo para 'arreglar' las imperfecciones." - "De forma súbita y sin pensarlo se venía de afuera de la mente la idea de volverme a frotar el rostro".

- "Luego esta fuerza interna activadora que activaba mi cuerpo se volvió involuntaria, y las sensaciones corporales aparecían sin que las piense".

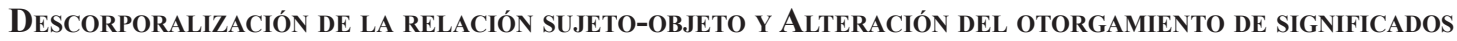

La descorporalización cambia la relación que el sujeto tiene con los objetos y hace que estos pierdan su utilidad ordinaria: los objetos se convierten en "inutilizables" pues aparecen como desprovistos de significado práctico. Las palabras ya no se utilizan para compartir significados de un mundo compartido, sino para crear un mundo alternativo, o un mundo por sí solo.
- La paciente utilizó con frecuencia el término "fuerza interna activadora" con un significado idiosincrático en el que se entremezclaban aspectos volitivos, cognitivos y de límites corporales. La abstracción de las palabras que se escapan de la situación hacia la cual ellas están referidas es una característica de la descorporalización de la relación sujeto-objeto.

\section{DESCORPORALIZACIÓN DE LA INTERSUBJETIVIDAD}

El cuerpo vivido transmite el conocimiento práctico de cómo interactuar con otros y de entender sus expresiones $\mathrm{y}$ acciones. Esto provee un pre-entendimiento fluido y automático de las situaciones de la vida diaria, lo cual permite conectar nuestro yo con el mundo a través de la habituación básica y la familiaridad. La alteración del sentido básico de "estar con otros" es remplazado por un sentido de separación, de imposibilidad de sintonización emocional, que puede convertirse en franca alienación, germen del autismo esquizofrénico.
- La paciente describió episodios de retraimiento social e incluso familiar que comenzaron tempranamente en su vida: "Cuando tenía 14 años, evitaba salir a la calle y solamente iba al colegio y a la iglesia”.

"En mi casa me sentía incómoda estando con mi familia, mejor me sentía cuando estaba a solas en mi cuarto”. cuerpo, y no ante cualquier otro (17). Cabe anotar que las alteraciones de la vivencia del self se han detectado clínicamente y mediante tests en estadios tempranos y hasta premórbidos de la esquizofrenia (14).

Se ha señalado que la corporalidad tiende a solaparse con el concepto de cuerpo vivido, puesto que para que el yo esté inmerso en el mundo no hay otra manera de lograrlo que siendo corpóreo (19). En ese sentido, la corporalidad se introduce en la semiología y en la nosología de la esquizofrenia a partir del concepto de cenestesia (20). El término cenestesia fue acuñado a partir de las raíces griegas 'koiné' (común) y 'aesthasis' (sensación) para designar aquellas sensaciones no dependientes de los órganos sensoriales sino provenientes de la percepción interna del propio cuerpo. Actualmente la cenestesia viene a ser la experiencia global que sintetiza todas las sensaciones corporales simples y en la cual se fundamenta la conciencia y la sensación de existencia de un yo. La prominencia de las cenestopatías y las aberraciones del esquema corporal son el rasgo esencial de la esquizofrenia cenestopática $(21,22)$. Su configuración como un subtipo de esquizofrenia fue recién planteada en 1957 por Gerd Huber. Actualmente, la esquizofrenia cenestopática se encuentra reconocida, pero no descrita, en el CIE-10, dentro del grupo de "otras esquizofrenias" y en el DSM-5 simplemente no se menciona a la esquizofrenia cenestopática como una entidad diagnóstica. Independientemente de si amerita constituir una nueva entidad nosológica, las perturbaciones de la cenestesia constituyen un importante tema de estudio dada su frecuencia en diferentes formas de esquizofrenia, especialmente en sus fases iniciales (12). Las cenestopatías son infrarreportadas, subdiagnosticadas y usualmente no son atendidas (23). 
Tabla 3. Alteraciones del cuerpo vivido y ejemplos ilustrativos del caso clínico. $(3,12)$

\begin{tabular}{|c|c|}
\hline \multicolumn{2}{|c|}{ Dinamización } \\
\hline $\begin{array}{l}\text { - Dinamización de los límites corporales: } \\
\text { Sensación de que fuerzas externas y extrañas } \\
\text { violan los límites corporales. } \\
\text { - Dinamización de la estructura corporal: las } \\
\text { partes del cuerpo mudan de posición. } \\
\text { - Externalización: partes o actividades del cuerpo } \\
\text { se localizan fuera de él. }\end{array}$ & $\begin{array}{l}\text { - La paciente experimentó la sensación de que sus actos } \\
\text { y sensaciones no le pertenecían y las sentía como si } \\
\text { vinieran de afuera. } \\
\text { - "Sentía que mi cabeza ya no estaba sobre mi cuello } \\
\text { sino encogida y dentro de mi pecho." }\end{array}$ \\
\hline \multicolumn{2}{|c|}{ OBJETIVACIÓN MÓRBIDA } \\
\hline $\begin{array}{l}\text { Funciones corporales normalmente tácitas se } \\
\text { vuelven ostensibles, funciones no espaciales se } \\
\text { ubican en el espacio, vivencia de "robotización". }\end{array}$ & $\begin{array}{l}\text { - "Sentía que mis pensamientos ahora estaban un poco } \\
\text { atrás de la cabeza, como por fuera del cerebro." }\end{array}$ \\
\hline \multicolumn{2}{|c|}{ FENÓMENOS DE TIPO DISMÓRFICO } \\
\hline $\begin{array}{l}\text { Cambios en la forma o apariencia del cuerpo, } \\
\text { usualmente hacia la deformidad o fealdad. }\end{array}$ & $\begin{array}{l}\text { - "Sentía que mis brazos estaban larguísimos y } \\
\text { también que todos mis dientes estaban tan juntos que } \\
\text { se apretaban." } \\
\text { - "De tanto arquear las cejas, mi cabeza cambió su } \\
\text { forma." }\end{array}$ \\
\hline \multicolumn{2}{|c|}{ VIVENCIAS DE TIPO DOLOROSO } \\
\hline $\begin{array}{l}\text { Paroxismos de dolor o vivencias corporales } \\
\text { inefables (cenestopatías) }\end{array}$ & $\begin{array}{l}\text { - "Mi boca me dolía porque mis dientes estaban todos } \\
\text { amontonados y parecía que reventarían unos contra } \\
\text { otros." }\end{array}$ \\
\hline
\end{tabular}

En el presente caso de esquizofrenia cenestopática, los fenómenos de descorporalización del yo, anomalías del otorgamiento de significados y descorporalización de la intersubjetividad se presentaron desde etapas tempranas de la enfermedad. Las cenestopatías, expresadas verbalmente de modo premioso y con predominio de las vivencias de elongación, agrandamiento y presión, se presentaron progresivamente a lo largo de la evolución de la enfermedad hasta ocupar el eje central de la vida de la paciente, causándole desmesurada preocupación que la conducía a ejecutar conductas extravagantes. Por otro lado, las alucinaciones auditivas y delusiones referenciales se limitaron a episodios esporádicos de poca prominencia y que aparecieron tardíamente en el curso del trastorno. El cuadro clínico de la paciente siguió una evolución tórpida que llegó incluso a poner en peligro su vida. Las alteraciones de la corporalidad y de la ipseidad quedan graficadas ampliamente en las vivencias patológicas de la paciente, todas ellas emblemáticas de la experiencia de un cuerpo vivido penosamente, hiperproséxicamente consciente de su distorsión, con agobio y pesadumbre, carente de "transparencia", torturado con cada movimiento psíquico de su yo poseedor.

El análisis del presente caso debe tener en cuenta que la paciente fue evaluada después de un tiempo de enfermedad de doce años. Esto pudo ocasionar que información de relevancia de la fase inicial de la enfermedad haya sido omitida por un sesgo de memoria. En nuestro caso se observa que los síntomas de descorporalización del yo se presentaron profusamente desde una fase temprana, como ha sido descrito en la bibliografía. Estos resultados respaldan la necesidad de ampliar la exploración de las experiencias subjetivas del paciente hacia áreas que no se tienen actualmente en cuenta en los manuales de clasificación de enfermedades mentales (10). Asimismo, dada la presentación de síntomas de descorporalización en 
etapas tempranas de la enfermedad, es de sumo interés realzar que investigaciones actuales están demostrando el beneficio de terapias basadas en la corporalidad (Body-oriented Psychotherapy; Dance and Movement Therapy) en la evolución temprana de la esquizofrenia, en cuanto a los disturbios de la descorporalización, pero asimismo registran prometedor efecto terapéutico sobre los síntomas negativos, lo que abre una novedosa perspectiva en el arsenal de tratamientos contra esta patología (24-26).

En conclusión, se puede observar que la esquizofrenia cenestopática presenta fenómenos de descorporalización y disturbios de la ipseidad desde estadios tempranos; estos desarreglos del self son característicos de la esquizofrenia -pero también de la esquizotipia- y permiten discriminar casos de trastorno bipolar; son asimismo predictores de la evolución a psicosis a partir de estadios clínicos de alto riesgo y se correlacionan estadísticamente con síntomas positivos y negativos, al igual que con síntomas de desorganización, lo que da una idea de su trascendencia y envergadura clínica -no solo psicopatológica- en el ámbito de la esquizofrenia (27). Los profesionales de salud mental deben conocer las características fenomenológicas de la esquizofrenia más allá de aquellas recogidas por los manuales de clasificación $(24,28)$, y así maximizar la oportunidad de identificar casos incipientes de esta enfermedad, con amplia comprensión fenomenológica de la vivencia individual, para poder brindar el beneficio de un tratamiento empático, integral y temprano.

Declaración de financiamiento: Autofinanciado.

Conflicto de intereses: Ningún conflicto por declarar.

\section{Correspondencia:}

Rubén Valle

Instituto Nacional de Salud Mental "Honorio

Delgado - Hideyo Noguchi”

Jr. Eloy Espinoza 709. Urb. Palao. San Martín de

Porres.

Lima 15102.

Correo electrónico: ruben_vr12@hotmail.com

Teléfono: 511-614 9200

\section{REFERENCIAS BIBLIOGRÁFICAS}

1. Pérez-Álvarez M. Las raíces de la psicopatología moderna: La melancolía y la esquizofrenia. Madrid: Pirámide; 2012.
2. Parnas J, Henriksen MG. Disordered self in the schizophrenia spectrum: a clinical and research perspective. Harv Rev Psychiatry. 2014; 22: 251-265.

3. Irarrázaval L. The lived body in schizophrenia: transition from basic self-disorders to full-blown psychosis. Front Psychiatry. 2015;6: 9. doi:10.3389/ fpsyt.2015.00009

4. Berrios GE, Marková IS. The self and psychiatry: a conceptual history. In: Kircher T, David A, editors. The Self in neuroscience and psychiatry. New York: Cambridge University Press; 2003.pp: 9-39.

5. Cermolacce M, Naudin J, Parnas J. The "minimal self" in psychopathology: re-examining the selfdisorders in the schizophrenia spectrum. Conscious Cogn. 2007; 16: 703-714.

6. Stanghellini G. Embodiment and schizophrenia. World Psychiatry. 2009; 8:56-9.

7. Fuchs T, Schlimme JE. Embodiment and psychopathology: a phenomenological perspective. Curr Opin Psychiatry. 2009; 22: 570-575.

8. Gallese V, Ferri F. Psychopathology of the bodily self and the brain: the case of schizophrenia. Psychopathology. 2014; 47: 357-364.

9. Doerr-Zegers O, Stanghellini G. Fenomenología de la corporalidad. Estudio de un caso paradigmático de esquizofrenia. Actas Esp Psiquiatr. 2015; 43:1-7.

10. Fuchs T. Corporealized and disembodied minds: a phenomenological view of the body in melancholia and schizophrenia. Philos Psychiatry Psychol. 2005;12:95-107.

11. Stanghellini G. Disembodied spirits and deanimated bodies: The psychopathology of common sense. New York: Oxford University Press; 2004.

12. Sass LA, Parnas J. Schizophrenia, Consciousness, and the Self. Schizophr Bull. 2003; 29:427-444.

13. Mancini M, Presenza S, Di Bernardo L, Lardo PP, Totaro S, Trisolini F, et al. The life-world of persons with schizophrenia. Journal of Psychopathology. 2014; 20: 423-434.

14. Parnas J, Moller P, Kircher T, Thalbitzer J, Jansson L, Handest P, et al. EASE: Examination of anomalous self-experience. Psychopathology. 2015; 38: 236258.

15. Urfer A. Phenomenology and psychopathology of schizophrenia: the views of Eugene Minkowski. Philos Psychiatry Psychol. 2001; 8: 279-289.

16. Borda JP, Sass LA. Phenomenology and neurobiology of self disorder in schizophrenia: primary factors. Schizophr Res. 2015; 169:464-473.

17. Giummarra MJ, Gibson SJ, Georgiou-Karistianis $\mathrm{N}$, Bradshaw JL. Mechanisms underlying embodiment, disembodiment and loss of embodiment. Neurosci Biobehav Rev. 2008; 32: 143-160.

18. Ardizzi M, Ambrosecchia M, Buratta L, Ferri F, Peciccia M, Donnari S, et al. Interoception and positive symptoms in schizophrenia. Front Hum Neurosci. 
2016; 10: 379. doi: 10.3389/fnhum.2016.00379

19. Legrand D. Phenomenological dimensions of bodily self-consciousness. In: Gallagher S, Editor. The Oxford Handbook of the Self. New York: Oxford University Press; 2011. pp. 204-227.

20. Rejón-Altable C. Extracto de la nada que cierra el círculo: apuntes para una psicopatología de la corporalidad. Átopos. 2009; 8: 49-66.

21. Röhricht F, Priebe S. Do cenesthesias and body image aberration characterize a subgroup in schizophrenia? Acta Psychiatr Scand. 2002; 105: 276-82.

22. Jenkins G, Röhricht F. From cenesthesias to cenesthopathic schizophrenia: a historical and phenomenological review. Psychopathology. 2007; 40: 361-368.

23. Blom JD, Sommer IEC. Hallucinations of bodily sensations. In: Blom JD, Sommer IEC. Hallucinations: Research and practice. New York: Springer; 2012. p. 157-169.

24. Sass LA. Self-disturbance and schizophrenia: structure, specificity, pathogenesis (current issues, new directions). Schizophr Res. 2014; 152:5-11.
25. Galbusera L, Fellin L, Fuchs T. Towards the recovery of a sense of self: An interpretative phenomenological analysis of patients' experience of body-oriented psychotherapy for schizophrenia. Psychother Res. 2017;0 :1-17. doi: 10.1080/10503307.2017.1321805

26. Martin LAL, Koch SC, Hirjak D, Fuchs T. Overcoming Disembodiment: The effect of Movement Therapy on negative symptoms in schizophrenia-A multicenter randomized controlled trial. Front Psychol. 2016; 7:483. doi: 10.3389/fpsyg.2016.00483

27. Henriksen MG, Nordgaard J. Self-Disorders in Schizophrenia. In: Stanghellini G, Aragona M, editors. An experiential approach to psychopathology. What is like to suffer from mental disorders? Switzerland: Springer; 2016. pp. 265-280.

28. Parnas J. A disappearing heritage: the clinical core of schizophrenia. Schizophr Bull. 2011; 37: 1121-1130.

Recibido: 17/05/2017

Aceptado: 11/09/2017 